\title{
CONTROVERSY
}

\section{Response to Dr Vikram Patel}

\section{SUMANT KHANNA}

The Psychiatric Clinic, 63 Paschimi Marg, Vasant Vihar, New Delhi 110057 INDIA e-mail: sumantk_2002@yahoo.co.in

Dr Patel questions the ethics of a placebo-controlled study in patients with acute mania and asks whether institutional review board approval of the trial had been obtained.

\section{Response}

The rationale for including a placebo group is that patients with mania generally show a high and variable placebo response, making it difficult to identify their responses to an active medication. By including a placebo group in this study, we were able to establish the efficacy and safety of risperidone over and above that observed with a placebo.

Placebo-controlled trials are valuable in that they expose the lowest number of patients to potentially ineffective treatments. In addition, inclusion of a placebo arm allows for a valid evaluation of adverse events attributable to treatment versus those independent of treatment. For these reasons, a placebo control in clinical studies is required or approved by key regulatory authorities.

The design and conduct of this placebo-controlled study were approved by an Independent Ethics Committee or Institutional Review Board at each of the eight study sites. Each patient was fully informed on the trial's procedure, including the information that he or she would be randomly assigned to receive an active drug (risperidone) or a placebo ("a tablet with no active medication"). Each patient or his/her legal representative provided informed consent and signed an informed consent form. As reported in the published article (page 229), "Signed informed consent was obtained for all participants and the study was conducted according to the Recommendations Guiding Physicians in Biomedical Research Involving Human Subjects, in the 1989 version of the Declaration of Helsinki (World Medical Association, 1989)."

Dr Patel questions how signed informed consent can be obtained from severely ill manic patients and suggests that the patients were kept in hospital "for longer than they possibly needed".

\section{Response}

It is our experience that severely ill patients are capable of giving their informed consent to participate in a trial. Capacity to consent is not automatically lost because of a symptom score on the YMRS scale. Hospitalisation until the achievement of symptom remission is part of ordinary clinical practice in India. In fact, Western health-care systems may care to emulate this practice.
Dr Patel suggests that a study of this design could potentially harm the patients.

\section{Response}

As expected, a high placebo response was shown by these hospitalised patients. Significant improvements versus baseline were seen on each of the efficacy measures in patients receiving placebo, as in the risperidone group: improvements in YMRS total scores at week-3 endpoint: $-10.5 \pm 1.3$ in patients receiving placebo and $-22.7 \pm 1.1$ in patients receiving risperidone ( $p$ $<0.001$ versus baseline in both groups); improvements in MADRS total scores at week-3 endpoint: $-2.5 \pm 0.3$ in placebo patients and $-3.2 \pm 0.4$ in risperidone patients $(p<0.001$ versus baseline in both groups); improvements in GAS scores at week3 endpoint: $12.9 \pm 1.7$ in placebo patients and $27.6 \pm 1.6$ in risperidone patients ( $p<0.001$ versus baseline in both groups); improvements in PANSS total scores at week-3 endpoint: $-5.7 \pm$ 1.2 in placebo patients and $-15.4 \pm 1.0$ in risperidone patients ( $p$ $<0.001$ versus baseline in both groups); clinical response $(\geq 50$ per cent reduction in YMRS score) at week-3 endpoint: in 36 per cent of placebo patients and 73 per cent of risperidone patients; and reductions in CGI-severity score at week-3 endpoint:-0.9 \pm 0.1 in placebo patients and $-2.0 \pm 0.1$ in risperidone patients ( $p$ $<0.001$ versus baseline in both groups).

Moreover, among the placebo patients, the proportion of patients whose severity of illness (CGI scale) was rated as "not ill," "mild," or "very mild" increased from 1 per cent at baseline to over one third (37 per cent) at endpoint (the increase was from 0 per cent to 72 per cent on the risperidone group).

Dr Patel suggests that haloperidol, a conventional antipsychotic, is "cheap, freely available, and constitutes usual care," and thus it was unnecessary to conduct a study of risperidone in these patients.

\section{Response}

As is well known, a primary disadvantage of the conventional antipsychotics is their association with adverse events, particularly extrapyramidal symptoms (EPS). In addition to improved safety, atypical antipsychotics have been shown to be as effective as or even more effective than conventional antipsychotics. Following are some results from three recent studies of an atypical antipsychotic compared with haloperidol:

Vieta et al, Br J Psychiatry, 2005: 347 patients with bipolar I disorder were randomised to aripiprazole or haloperidol. Prevalence of EPS: in 63 per cent of haloperidol patients versus 
24 per cent of aripiprazole patients. Proportion of treatment responders (50 per cent improvement in YMRS scores): 50 per cent of aripiprazole patients versus 28 per cent of haloperidol patients $(P<0.001)$.

McIntyre et al, Eur Neuropsychopharmacol, 2005: 303 patients with bipolar I disorder were randomised to quetiapine, haloperidol, or placebo. Prevalence of EPS: in 60 per cent of haloperidol patients, 13 per cent of quetiapine patients, and 16 per cent of placebo patients. Improvements in YMRS scores were significantly greater in patients receiving quetiapine or haloperidol than placebo $(\mathrm{P}<0.001)$.

Smulevich et al, Eur Neuropsychopharmacol, 2005: 438 patients randomised to risperidone, haloperidol, or placebo. Prevalence of EPS: in 85 per cent of haloperidol patients, 34 per cent of risperidone patients, and 18 per cent of placebo patients. Improvements in YMRS scores were significantly greater in patients receiving risperidone or haloperidol than placebo
$(\mathrm{P}<0.001)$.

Haloperidol and other older antipsychotics have been known to increase depressive symptoms and the risk for provoking patients into the depressive phase of bipolar illness. Depressive symptoms are debilitating and can increase the risk for mortality. Atypical antipsychotics such as risperidone are not known to worsen depressive symptoms.

Dr Patel writes that "the majority of patients (>80 per cent) were already receiving psychotropics at the time of enrollment (but) these effective treatments were discontinued as a prerequisite for participation in the trial ... (the patients) were actually deliberately stopped from receiving such treatment." Dr. Patel seems to have overlooked the fact that the patients had been hospitalised for the treatment of acute mania, indicating surely that their current "cheap and effective" treatments were not controlling their symptoms.

\section{Thank you, reviewers}

All submissions to the journal undergo an extensive review by internal reviewers from within the journal's editorial boards as well as external peer reviewers. We would like to thank all our editorial board reviewers, and also name the following external experts who have reviewed articles for the journal during 2005:

Rani Chintam, Sharad Deshpande, Gopa Kumar, K Mathiharan, JP Muliyil, Laxmi Murthy, M R Hariharan Nair, Samiran Nundy, Maya Parihar, Prathima Radhakrishnan, Ravi Ramakanthan, Karuna Ramesh, Satyajit Rath, Anita Roy, Peush Sahni, Chinu Srinivasan, Avinash Supe, Prathap Tharyan, Joe Verghese. 